\title{
Optical observations of IGR J00291+5934 in the post outburst phase
}

\author{
M. T. Reynolds ${ }^{a}$, P. Elebert ${ }^{a}$, P. J. Callanan ${ }^{a}$, B. Field ${ }^{a}$, P. Tuite $^{a}$, \\ M. A. P Torres $^{b}$, D. Steeghs ${ }^{b}$, P. M. Garnavich ${ }^{c}$, D. M. Terndrup ${ }^{d}$, \\ A. V. Filippenko ${ }^{e}$, R. J. Foley ${ }^{e}$ \& E. T. Harlaftis ${ }^{f}$ \\ ${ }^{a}$ Department of Physics, University College Cork, Ireland \\ email: m.reynolds@ucc.ie \\ ${ }^{b}$ Center for Astrophysics, 60 Garden Street, Cambridge, MA 02138, USA \\ ${ }^{c}$ Department of Physics, University of Notre Dame, Notre Dame, IN 46556-5670, USA \\ ${ }^{d}$ Department of Astronomy, The Ohio State University, Columbus, OH 43210, USA \\ ${ }^{e}$ Astronomy Department, University of California, Berkeley, California 94720, USA \\ ${ }^{f}$ National Observatory of Athens, PO Box 20048, Athens 11810, Greece
}

\begin{abstract}
We present optical observations of the newly discovered accretion powered millisecond pulsar IGR J00291+5934, undertaken in the weeks following its outburst on $2^{\text {nd }}$ December 2004. The decay to quiescence is seen to be highly variable with no indication of a modulation on the $\sim 2.46 \mathrm{hr}$ orbital period apparent in the data, consistent with a system at low inclination. We also have a single Keck LRIS spectrum of the companion to IGR J00291+5934 taken 10 days after outburst. Strong hydrogen and helium emission lines are observed confirming the identity of the counterpart.
\end{abstract}

Keywords. X-rays: binaries, pulsars: individual (IGR J00291+5934).

\section{Introduction}

IGR J00291+5934 was discovered by INTEGRAL on 2004 December 2 nd (Shaw et al. 2005). The optical counterpart was identified two days later and was found to have a magnitude $\mathrm{R} \approx 17.4$ (Fox et al. 2004). It was quickly identified as the 6 th member of the class of accretion powered millisecond X-ray pulsars to be discovered. It has the fastest spin period yet observed in this class $\mathrm{p}_{\text {spin }} \sim 1.7 \mathrm{~ms}$ and the second longest orbital period $\sim 2.46 \mathrm{hr}$. We present here the first optical study of its outburst and decay.

\section{Photometry}

Two nights of R-band data were obtained on 2004 December 9th and 18th at the $1.3 \mathrm{~m}$ MDM telescope at KittPeak and the 4m WIYN telescope respectively (see Fig. 1). We also obtained 2 nights of white light observations from the $1.2 \mathrm{~m}$ telescope at the Kryoneri observatory in Greece. The resulting lightcurves were phased to the ephemeris of Markwardt et al. (2004). The lightcurves are observed to be highly variable with no significant modulation at the orbital period evident (Fig. 1). The observed fading is consistent with that observed by Bikmaev et al. (2005). The IR counterpart was first observed on December 8th (see Steeghs et al., 2004), 6 days after the onset of the outburst.

\section{Spectroscopy}

In an effort to confirm the identification by Roelofs et al. (2004), a single 300s LRIS spectrum of the proposed optical counterpart was obtained 10 days post outburst (Filippenko et al., 2004), Fig. 1. We observe broad (FWHM $=1200 \mathrm{~km} / \mathrm{s}$ ) emission lines of $\mathrm{H} \alpha 656 \mathrm{~nm}, \mathrm{H} \beta 486 \mathrm{~nm}$, and $\mathrm{HeI} 667.8 \mathrm{~nm}$, as well as narrow (FWHM = $300 \mathrm{~km} / \mathrm{s}$ ), 

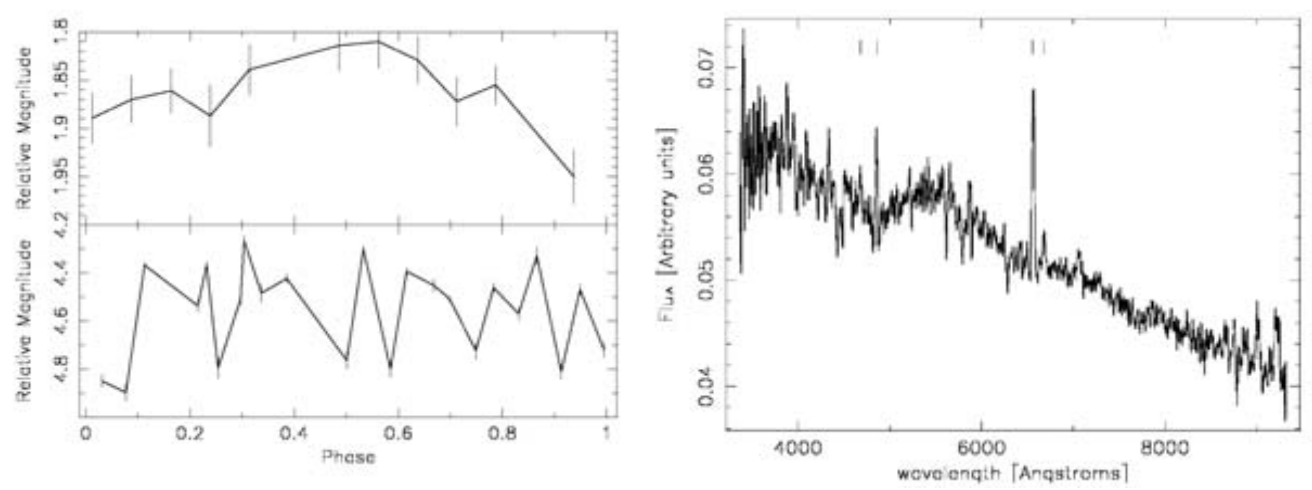

Figure 1. LeFT: The R-band light curve. The top panel is from 9.12 .04 with the bottom panel obtained 9 days later. RIGHT: Keck LRIS spectrum of the optical counterpart taken on 12.12.04. The markers indicate, from left to right, the positions of the HeII $468.6 \mathrm{~nm}(\mathrm{EW}=0.06 \mathrm{~nm})$, $H \beta 486 \mathrm{~nm}(\mathrm{EW}=0.54 \mathrm{~nm}), H \alpha 656 \mathrm{~nm}(\mathrm{EW}=0.96 \mathrm{~nm}) \&$ HeI lines $667.8 \mathrm{~nm}(\mathrm{EW}=$ $0.1 \mathrm{~nm})$.

very weak HeII $468.6 \mathrm{~nm}$ emission. The lines are superposed on a blue continuum. These are classic hallmarks of an X-ray transient in outburst and hence they firmly establish the counterpart of the accreting X-ray pulsar.

\section{Discussion}

IGR J00291+5934 is the 6 th member of the class of accretion powered millisecond $\mathrm{X}$-ray pulsars to be discovered and the third 'long' orbital period system. The decay of $I G R$ J00291+5934 is the fastest yet observed in this class of binary, with an efolding time $\tau_{e}=5.5$ days. In comparison, the e-folding time for $S A X J 1808.4-3658$ was $\sim 14$ days. This may be due to the relatively small accretion disk present in these systems, because of (i) the short orbital period (and small system size), and (ii) the truncated inner disk due to the magnetosphere of the neutron star: this is similar to what happens during outbursts of intermediate polars (Angelini et al., 1989). Recently Burderi et al. (2005) suggested that IGR J00291+5934 could be a high inclination system; however the absence of any clear modulation in our data is inconsistent with this.

\section{Acknowledgements}

The data presented in this paper was obtained at the following observatories; WIYN, MDM, Kryoneri, PAIRITEL \& Keck I.

MTR, PE \& PJC wish to acknowledge financial support from Science Foundation Ireland. The authors wish to dedicate this paper to the memory of Emilios T. Harlaftis, who passed away in tragic circumstances earlier this year.

\section{References}

Angelini, L. \& Verbunt, F., 1989, MNRAS, 238, 697

Bikmaev, I., Suleimanov, V., Galeev, A. \& 7 co-authors, 2005, ATel, 395

Burderi, L., Di Salvo, T., Riggio, A. \& 6 co-authors, astro-ph/0509224

Filippenko, A.V., Foley, R.J. \& Callanan, P.J., 2004, ATel, 366

Fox, D.B. \& Kulkarni, S.R., 2004, ATel, 354

Markwardt, C.B., Galloway, D.K., Chakrabarty, D. \& 2 co-authors, 2004, ATel, 360

Roelofs, G., Jonker, J.G., Steeghs, D., Torres, M.A.P. \& Nelemans, G., 2004, ATel, 356

Shaw, S.E., Mowlawi, N., Rodriguez, J., Ubertini, P. \& 7 co-authors, astro-ph/0501507

Steeghs, D., Blake, C., Bloom, J.S., Torres, M.A.P., Jonker, P.G., \& Starr, D., 2004, ATel, 363

Vanderspek, R., Morgan, E., Crew, G., Graziani, C., \& Suzuki, M., 2005, ATel, 516 